\title{
Isolation and Ex Vivo Expansion of Human Hematopoietic Stem Cells Derived from Umbilical Cord Blood
}

\author{
Mao Pang1, Shiqian Han², Meng Ren1, Dongwei Guan', Tianying Kuang1, Shuixia Liu, \\ Yesong Liu' ${ }^{4}$, Jieping Chen ${ }^{5}$, Fengchao Wang6, Chunmeng Shi ${ }^{7}$, Zhijia Ye1,2*
}

${ }^{1}$ Laboratory Animal Research Center, Chongqing University School of Medicine, Chongqing, China

${ }^{2}$ Department of Tropical Medicine, Third Military Medical University, Chongqing, China

${ }^{3}$ Cancer Center, Daping Hospital, Third Military Medical Center, Chongqing, China

${ }^{4}$ Cornell University, Ithaca, USA

${ }^{5}$ Department of Hematology, Southwest Hospital, Third Military Medical University, Chongqing, China

${ }^{6}$ State Key Laboratory of Trauma, Burns and Combined Injury, Institute of Combined Injury, Third Military Medical University, Chongqing, China

${ }^{7}$ State Key Laboratory of Trauma, Burns and Combined Injury, Institute of Rocket Force Medicine, Third Military Medical University, Chongqing, China

Email: *yezj918@cqu.edu.cn

How to cite this paper: Pang, M., Han, S.Q., Ren, M., Guan, D.W., Kuang, T.Y., Liu, S.X., Liu, Y.S., Chen, J.P., Wang, F.C., Shi, C.M. and Ye, Z.J. (2021) Isolation and EX Vivo Expansion of Human Hematopoietic Stem Cells Derived from Umbilical Cord Blood. Stem Cell Discovery, 11, 1-13. https://doi.org/10.4236/scd.2021.111001

Received: January 2, 2021

Accepted: January 27, 2021

Published: January 30, 2021

Copyright ( 2021 by author(s) and Scientific Research Publishing Inc. This work is licensed under the Creative Commons Attribution International License (CC BY 4.0).

http://creativecommons.org/licenses/by/4.0/ (c) (i) Open Access

\begin{abstract}
Umbilical cord blood (UCB) is a current major source of hematopoietic stem cells (HSCs) for cell transplantation therapy. Cell transplantation with HSCs derived from UCB is advantageous over transplantation with HSCs from adult tissues. However, the low number of HSC derived from a single unit of UCB limits its application. Thus, ex vivo expansion is a good option to create more UCB HSCs for clinical application. The strategies for HSC expansion in vitro focus on mimicking the composition and structure of HSC natural niche by enhancing self-renewal and inhibiting lineage differentiation of HSCs. In the past decade, the mechanisms of the interaction between HSC and the natural niche have been deeply investigated. This great progress in basic research has led to advancements in UCB HSC ex vivo expansion. In addition, the biological characteristics of the originally isolated UCB HSCs correlate with outcome of subsequent ex vivo expansion. In this paper, we summarize the late progress achieved in isolation and ex vivo expansion of UCB HSCs. Importantly, we attempt to provide an impact and practicable procedure to expand UCB HSC in vitro from isolation of original HSCs to identification of expanded HSCs.
\end{abstract}

\section{Keywords}

Hematopoietic Stem Cells, Isolation, Ex Vivo Expansion 


\section{Introduction}

Transplantation of hematopoietic stem cells is an important curative therapy for hematologic malignancies and nonmalignant hematologic disorders [1]. UCB has been established as a major source of HSCs for cell transplantation-based therapies [2] [3]. Compared to HSCs from adult donors, UCB HSCs are more advantageous as they have: less stringent HLA matching requirements, lower risk of graft-versus-host disease (GVHD) [4], lower donor-recipient viral transmission risk, lower relapse rate, and higher survival rate. However, transplantation using HSCs derived from UCB has only been applied in pediatric patients due to the limited number of HSC obtained from a single unit of UCB [5] [6]. Attempts to expand UCB HSC in order to enable its applicability in all patients are still continuing.

A comprehensive procedure for UCB HSC expansion includes: UCB collection, HSC isolation, HSC ex vivo expansion and character identification of expanded HSC. The biological characteristics of the obtained HSCs are critical in determining the outcome characteristics of the ex vivo expanded HSCs [7] [8]. UCB collection and HSC isolation procedure affect the biological activity of unexpanded HSCs. UCB collection was suggested to carry out on delivery when the placenta is still unexpelled, the uterine paroxysmal contraction pressure increases volume of the collected cord blood [9]. The intermittent and gentle shaking of the blood bag during the collection process can prevent the formation of blood clots [9] [10]. Cyclosporine A (CSA), an inhibitor of reactive oxygen species (ROS), is used to maintain renewal property and inhibit lineage differentiation of the HSCs by ameliorating oxygen shock/stress (EPHOSS) [1].

Human HSCs have the capacity of massive self-renewal division in vivo [11] [12]. The robust in vivo expansion of HSC was demonstrated by HSC transplantation. However, HSC ex vivo expansion still encounters many challenges. HSC expansion in vivo is strictly controlled by HSC niche [11] [13]. The strategies for HSC ex vivo expansion aimed to mimic HSC niche in vivo [14] [15]. But the knowledge about composition and structure of HSC niche in vivo remains limited. The cell growth factors and cell development factors used in HSC ex vivo expansion were successfully identified from HSC niche cells [8] [16] [17] [18]. However, the cocktail of those protein factors was proclaimed to not support HSC self-renewal ex vivo [19]. This conclusion indicated that HSC self-renewal not only depends on the growth and development factors but also depends on the clues out of the protein factors. In the past ten years, chemical library screening based on HSC biological characteristics has identified some useful chemical compounds in HSC ex vivo expansion [20]. Among of those chemicals, SR-1 and UM171 have great potential in maintaining biological characters of expanded HSCs through their synergistic effects with the growth factor panel (SCF, TPO, Flt3-ligand) [19] [20]. The optimized recipe with combination of the two chemicals and the panel of growth factors was shown to expand HSC more than 30 folds in effective engraftment [20]. The expanded HSCs by SR-1 and 
UM171 have entered clinical trials [21] [22]. For energy metabolism, HSCs prefer glycolysis over oxidative phosphorylation (OXPH) [23]. A low concentration of calcium was demonstrated to enhance HSC expansion in vitro through maintaining glycolysis in HSCs [24]. Growth factors and chemicals are mostly applied in HSC expansion procedures. Mathematical models were benefit for giving a meaning to guide the optimization of composition and concentration of the growth factors and chemicals. Here we summarize the updated procedures applied in the isolation and ex vivo expansion of UCB HSCs.

\section{Methods}

\subsection{Isolation of Cord Blood-Derived Hematopoietic Stem Cells}

\subsubsection{Collection of the Umbilical Cord Blood}

\section{Materials}

$70 \%$ Alcohol;

Iodine;

Disposable blood collection bag (200 mL, NIGALE);

Ice box (FYL-BW-11L, FuYi electric).

\section{Procedure}

An informed consent was sought from patients before procedure commencement.

1) Record obstetric and family medical history, exclude etiology and genetic anomalies.

2) Cut the cord while the placenta is still in utero upon delivery of the term baby. Use $70 \%$ alcohol and iodine to sterilize the umbilical cord, puncture the umbilical vein, then collect UCB by the intermittent contraction of the uterus [9]. Allow continuous gentle shaking of the blood collection bag until no more blood flows out of the cord. Close the blood collection bag and place it in the icebox.

3) Collect at least $60 \mathrm{mls}$ of the cord blood. This contains more than $10^{8}$ mononuclear cells [10]. Do not allow blood clots to form. Transport the cord blood in icebox immediately to the laboratory. HSC isolation should start within 2 hours.

\subsubsection{Isolation of Human CD34 Positive Cell}

\section{Materials}

1) Phosphate buffered saline pH7.2 (PBS) (SH30256.01, HyClone).

2) IMDM Modified (SH30228.01, HyClone).

3) CSA (1101, R\&D).

4) Ficoll-Paque PLUS (17-1440-02, GE).

5) EasySep Human Cord Blood CD34 positive selection kit II (17897, STEMCELL Technologies).

6) Biosafety cabinet.

7) Multichannel pipettes, Tips, Pasteur pipettes.

\section{Procedure}


1) Prepare Ficoll-Paque PLUS and umbilical cord blood at room temperature. Dilute the CSA to a final concentration of $50 \mu \mathrm{g} / \mathrm{mL}$ in PBS and label as solution A [1].

2) Dilute the umbilical cord blood in solution $A$ at a ratio of 1:1.

3) Use a pipette to transfer $15 \mathrm{~mL}$ Ficoll to a sterile $50 \mathrm{~mL}$ centrifuge tube. Carefully layer the diluted blood sample $(30 \mathrm{~mL})$ on Ficoll-Paque PLUS.

4) Centrifuge the diluted blood sample at $400 \mathrm{~g}$ for 30 minutes at room temperature, the centrifuge needs to turn off the brake. Gently remove the sample and avoid mixing of the separated liquid layers.

5) Use a Pasteur pipette to transfer the lymphocyte layer to a sterile $50 \mathrm{~mL}$ centrifuge tube. Add at least 3 volumes of IMDM to the lymphocytes containing tube. Re-centrifuge at $500 \mathrm{~g}$ for 10 minutes at room temperature and remove the supernatant.

6) Suspend the lymphocytes in IMDM by gently drawing them in and out of the Pasteur pipette. Centrifuge again at $200 \mathrm{~g}$ for 10 minutes at room temperature, remove the supernatant, and re-suspend the cell pellet in IMDM. Then centrifuge at $400 \mathrm{~g}$ for 10 minutes at room temperature.

7) Isolate the stem cells using a CD34 positive human cord blood selection kit according to the manufacturer's instructions. All solutions for isolation contain CSA at a concentration of $50 \mu \mathrm{g} / \mathrm{mL}$ [1].

\subsubsection{Phenotypic Characterization of Isolated CD34+ Cells \\ Flow Cytometry Analysis of Phenotype \\ Materials}

1) Human hematopoietic stem cell.

2) PBS pH7.2.

3) Blocking buffer: $2 \%(\mathrm{v} / \mathrm{v})$ mouse serum in PBS.

4) Dilution buffer: $2 \%(\mathrm{v} / \mathrm{v})$ fetal bovine serum (FBS) in PBS.

5) PE-labeled anti-human CD34 (12-0349-42, eBioscience).

6) PE-Cy7 labelled anti-human CD45RA (25-0458-42, eBioscience).

7) PE-Cy5 labelled anti-human CD49f (551129, BD Biosciences).

8) APC labelled anti-human CD90 (559869, BD Biosciences).

9) FTIC labelled anti-human CD38 (11-0388-42, eBioscience).

10) BD FACSVerse flow cytometer, FlowJo software.

\section{Procedure}

1) Harvest the stem cells, centrifuge at $300 \mathrm{~g}$ for 5 minutes at room temperature, and keep the cell pellet.

2) Resuspend the cells in cold PBS, perform cell count using trypan blue andhemacytometer. The number of cells in each sample is approximately $2-5 \times 10^{5}$.

3) Add blocking buffer, incubate the cells at $4^{\circ} \mathrm{C}$ for 30 minutes. Then centrifuge at $300 \mathrm{~g}$ for 5 minutes at $4^{\circ} \mathrm{C}$ and discard the supernatant. Suspend the cells in a dilution buffer, $100 \mu \mathrm{L} /$ sample.

4) Cells are stained with the following antibodies according to the manufacturer's instructions: PE-labelled anti-human CD34, PE-Cy7 labelled anti-human 
CD45RA, PE-Cy5 labelled anti-human CD49f, APC labelled anti-human CD90, FTIC labelled anti-human $\mathrm{CD} 38$. Incubate the cells at $4^{\circ} \mathrm{C}$ for 30 minutes in dark [20].

5) Centrifuge at $300 \mathrm{~g}$ for 5 minutes at $4^{\circ} \mathrm{C}$. Wash the cells with PBS for $2-3$ times. Suspend the cells in PBS.

6) Put the cells on a shaker before reading at the flow cytometer. The human cold blood stem cells express $\mathrm{CD} 34^{+} \mathrm{CD} 38^{-} \mathrm{CD} 45 \mathrm{RA}^{-} \mathrm{CD} 90^{+} \mathrm{CD} 49 \mathrm{f}^{+}$phenotype.

\subsubsection{Wright-Giemsa Stain}

\section{Materials}

1) Wright-Giemsa solution (G1020, Solarbio).

2) Phosphate buffer pH6.4 [0.01 M].

3) Inverted microscope (Olympus).

4) Thin-layer Cell Preparation System (A78300003, ThermoFisher).

\section{Procedure}

1) Resuspend the cell pellet in $2 \%$ FBS in PBS per $1 \times 10^{5}$ cells, to a final volume of $300 \mu \mathrm{L}$.

2) Assemble the accessories of the thin-layer cell preparation system, clamp the slides under the specimen chamber. Immediately transfer cell suspension into the specimen chamber. Centrifuge at $1000 \mathrm{rpm}$ for 5 minutes, dry in air.

3) Fix cells with $4 \%$ formaldehyde solution for 10 minutes and rinse the cells with distilled water to remove residual formaldehyde.

4) Stain cells with Wright-Giemsa solution for 1 - 2 minutes. Add Phosphate buffer pH6.4 [0.01 M] which equal volume to Wright-Giemsa solution, mix well, and stain for another 3 - 5 minutes.

5) Rinse cells three times with distilled water, use a filter paper to absorb the remaining liquid. Visualize the images under a light microscope.

\subsection{Expansion of UCB HSC}

\subsubsection{Preparation of Basic Media}

A low concentration of calcium $\left(0.02 \mathrm{mM} \mathrm{CaCl}_{2}\right)$ can enhance the function of hematopoietic stem cells and maintain the biological activity. StemSpan media (Stem cell biotechnology) which contains human growth factors and cytokines, is widely used in the expansion of human HSCs. These growth factors are supplemented in a basic media containing a high concentration of $\mathrm{CaCl}_{2}(1.6 \mathrm{mM})$. The basic medium with a low concentration of calcium can be generated by the following procedure [24].

\section{Materials}

1) $\mathrm{Ca}^{2+}$-freeDMEM (21068028, Gibco).

2) StemSpan CD34+ Expansion Supplement (10X) (02691, STEMCELL technologies).

3) StemPro-34 SFM Kit (10639011, Thermo Fisher).

4) L-Glutamine (25030081, Gibco).

5) Sodium Pyruvate (11360070, Gibco). 
6) HEPES (15630106, Gibco).

\section{Procedure}

1) Using $\mathrm{Ca}^{2+}$-free DMEM as the basic media reconstitute with $10 \mathrm{mM}$ HEPES, $1 \times$ Penicillin/Streptomycin Solution, $1 \times$ L-Glutamine, $1 \mathrm{mM}$ Sodium Pyruvate.

2) Supplement with $1 \mathrm{x}$ Nutrient supplement (StemPro ${ }^{\mathrm{rm}}-34$ SFM Kit) to provide serum-free components, and $1 \times$ StemSpan CD34+ Expansion Supplement to provide human cytokines [24].

3) Preparea completestock media containing $2 \mathrm{mM} \mathrm{CaCl}_{2}$, filter with $0.22 \mu \mathrm{m}$ disposable filter, and store the filtrate at $4{ }^{\circ} \mathrm{C}$. Dilute the $\mathrm{CaCl}_{2}$ stock solution to a final concentration of $0.02 \mathrm{mM}$ to obtain a low concentration of calcium.

\subsubsection{Optimize the Concentration of Cytokines}

Many types of cytokines have been demonstrated to stimulate in vitro HSC expansion [22] [24]. However, the expansion effects with a combination of cytokines and the optimized cytokines' concentration have not yet been fully determined. Thus, optimization of combinations and concentration of cytokines is important for efficient HSC expansion. An efficient and reliable mathematical model can benefit for the optimization process.

Response Surface Methodology (RSM) [25] is often used to solve the multi-objective optimization problem in biomedicine [26] [27] [28] [29]. RSM is a collection of mathematical and statistical techniques that are useful for modeling and analysis of problems in which a response of interest is influenced by several quantifiable variables (or factors) [25] [30]. RSM is sequential, determining the significant factor variables, gradually eliminating the non-significant factors, and determining the optimal distance of the response value [25]. If the existing conditions are not optimal, a series of adjustments can give the optimal direction. The common method of RSM is the face-centered cube design (FC-CD) [25]. This model design only needs three levels of each factor: $-1,+1,0$, the model design is cube [25] [31] [32].

We give an example for optimizing the concentrations of 3 growth factors using a face-centered cube design (FC-CD), the growth factors include stem cell factor (SCF), thrombopoietin (TPO) and FMS-like tyrosinekinase 3 ligand (Flt3L).

\section{Materials}

1) Stemspan SFEM (09600, STEMCELL technologies).

2) Human recombinant SCF (78062, STEMCELL technologies).

3) Human recombinant TPO (78210, STEMCELL technologies).

4) Human recombinant Flt3/Flk-2 Ligand (78009, STEMCELL technologies).

\section{Procedure}

1) Using StemSpan SFEM media culture HSC, and seed cells at the concentration of $3 \times 10^{4} / \mathrm{mL}$.

2) Design for optimization of a cocktailof cytokines. SCF, Flt-3L and TPO which are tested either at low concentration $(-1,0 \mathrm{ng} / \mathrm{mL})$, medium concentra- 
tion $(0,50 \mathrm{ng} / \mathrm{mL})$, or high concentration $(+1,100 \mathrm{ng} / \mathrm{mL})$. The experimental design (Figure 1) has 8 factorial points, 6 axial points, 3 replicated center points (which improve the analysis accuracy), and a total of 17 runs as shown in Table 1 [25] [30].

3) Carefully transfer $\mathrm{HSC}$ to a $37^{\circ} \mathrm{C}, 5 \%$ supplemented $\mathrm{CO}_{2}$ incubator. On day 7 , perform cell count and flow cytometry analysis of CD34 positive cells.

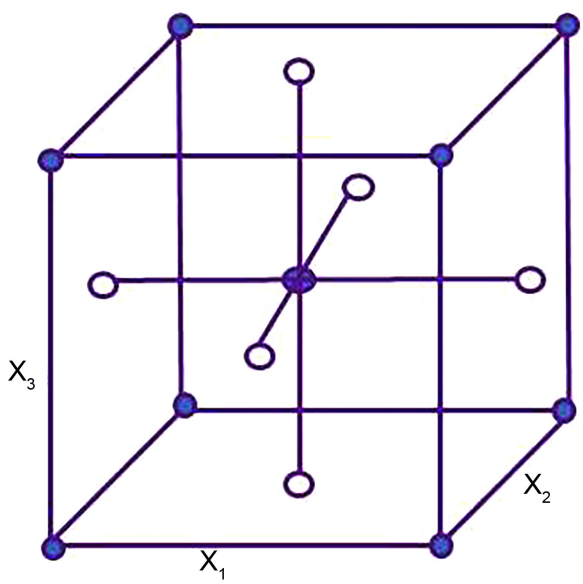

Figure 1. Experiments design model for 3 factors. The diagram was modified from the reference: Montgomery DC (2017).

Table 1. Design for optimization of the cytokines cocktail.

\begin{tabular}{|c|c|c|c|}
\hline Runs & SCF & Flt3-L & TPO \\
\hline 1 & -1 & -1 & 1 \\
\hline 2 & -1 & 1 & 1 \\
\hline 3 & 1 & -1 & 1 \\
\hline 4 & 1 & 1 & 1 \\
\hline 5 & 1 & 1 & -1 \\
\hline 6 & 1 & -1 & -1 \\
\hline 7 & -1 & 1 & -1 \\
\hline 8 & -1 & -1 & -1 \\
\hline 9 & 0 & 0 & 1 \\
\hline 10 & 0 & 0 & -1 \\
\hline 11 & -1 & 0 & 0 \\
\hline 12 & 1 & 0 & 0 \\
\hline 13 & 0 & 1 & 0 \\
\hline 14 & 0 & -1 & 0 \\
\hline 15 & 0 & 0 & 0 \\
\hline 16 & 0 & 0 & 0 \\
\hline 17 & 0 & 0 & 0 \\
\hline
\end{tabular}


4) The expanded cells of each sample are cultured on methylcellulose media for colony-formingunit (CFU) assay.

5) Calculate and record every experiment FI (fold increase) total nucleated cells (TNC), FI CD34+, FI BFU-E, FI CFU-GM, FI CFU-GEMM, and FI CFU-mix as the expansion response. Perform statistical analysis usingDesign-Expert software and SAS. A p-value of all variables in the model of less than or equal to 0.05 , is considered statistically significant. Finally, determine the optimal concentration range of SCF, TPO, Flt3-L and carry out the verification process [25].

\subsubsection{Optimizing Concentration of the Chemical Compounds}

SR-1 and UM171 are powerful chemical molecules screened from chemical library containing more than 5000 compounds [19] [20]. SR-1 is an antagonist of the aryl hydrocarbon receptor (AhR). It inhibit the AhR signaling pathway and promote the proliferation of HSC by binding AhR to prevent its binding to AhR photoaffinity ligand (PAL) [19] [21]. Conversely, UM171 enhance the human HSC self-renewal machinery independently of AhR suppression [20], whereas induce a rheostatic regulation of inflammatory and anti-inflammation/detoxification programs [33]. UM171 greatly reduces the risk of long-term frequent immune complications (GVHD) [22]. But SR-1 or UM171 could not stimulate HSC proliferation alone [19] [20]. Meanwhile, the growth factor panel can prime HSC number increase but cannot inhibit HSC lineage differentiation [16] [20]. The combination of growth factors and chemical compounds achieved great progress in HSC ex vivo expansion [19] [20] [34]. Optimization of chemical concentrations used in the culture medium formula containing the growth factor panel is necessary. FC-CD model is a helpful tool to guide the procedure optimization.

\section{Materials}

1) Stemspan SFEM(09600, STEMCELL technologies).

2) Human recombinant SCF (78062, STEMCELL technologies).

3) Human recombinant TPO (78210, STEMCELL technologies).

4) Human recombinant Flt3/Flk-2 Ligand (78009, STEMCELL technologies)

5) SR-1 (72342, STEMCELL technologies).

6) UM171 (72914, STEMCELL technologies).

\section{Procedure}

1) Prepare StemSpan SFEM media with $50 \mathrm{ng} / \mathrm{mL}$ TPO, $100 \mathrm{ng} / \mathrm{mL}$ SCF, 100 $\mathrm{ng} / \mathrm{mL}$ Flt3-L, $1 \times$ Penicillin/Streptomycin Solution.

2) Seed cells at a concentration of $3 \times 10^{4} / \mathrm{mL}$.

3) Design for optimization of the compounds. UM171 and SR-1are tested either at a low level ( -1 , low concentration), middle level (0, average of low and high concentrations), high level ( +1 , high concentration). The experimental design as shown in Figure 2 is composed of 4 factorial points, 4 axial points, 2 replicated center points with a total of 10 runs. [25]

4) Record every experiment FI TNC, FI CD34+, FI BFU-E, FI CFU-GM, FI CFU-GEMM, and FI CFU-mix. 


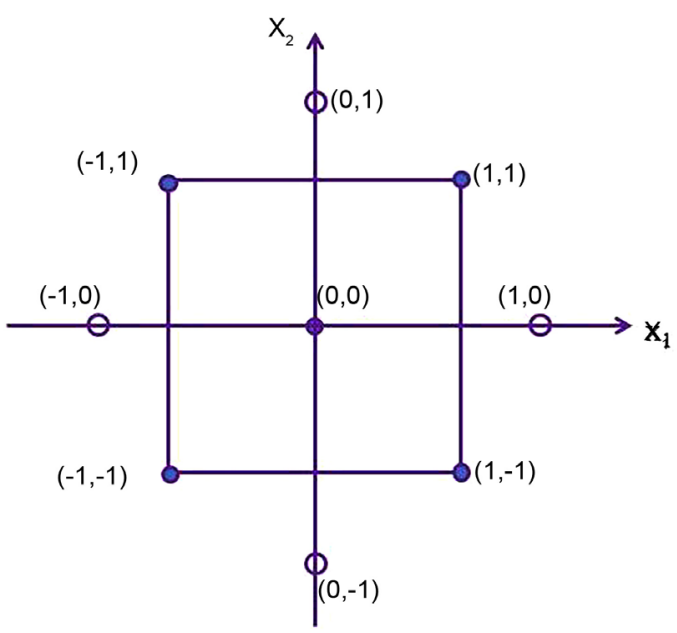

Figure 2. Experiments design model for 2 factors. The diagram was modified from the reference: Montgomery DC (2017).

\subsubsection{HSC Expansion with a Bioreactor}

A bioreactor system with fed-batch function can benefit for HSC ex vivo expansion by continously providing with fresh medium and removing cell metabolites. The fed-batch culture system can keep HSC growth in a stable culture environment [20] [35]. The brands of cell culture bags approved by FDA have found a use in fed-batch culture [35]. The cell culture bag made of fluoroethyl polymer(FEP) is gas permeable and water impermeable. A bag loaded with the initial HSCs suspension is put on a shaker in cell culture incubator and connected to a pump-loaded syringe system (Figure 3). The pump is set up to continously deliver the culture medium into the cell bag with a designed flow rate [20] [35].

\subsection{Function Analysis of Expanded HSC in Vitro-Clone Forming Unit (CFU) Assay}

\section{Materials}

1) IMDM (Hyclone).

2) FBS (Gibco).

3) MethoCult H4034 Optimum (04034, STEMCELL technologies).

\section{Procedure}

1) Prepare medium, IMDM with $2 \%$ FBS.

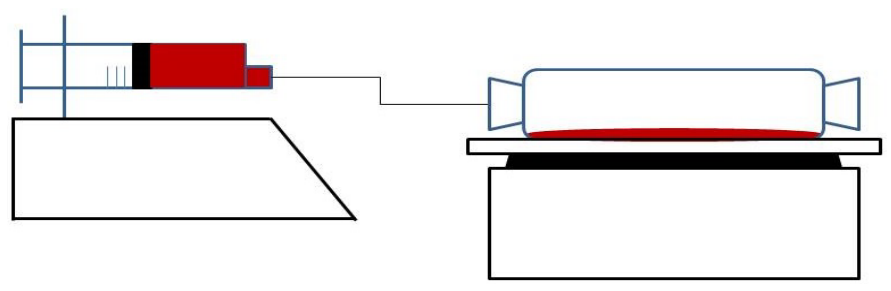

Figure 3. Fed-batch culture system diagram. The diagram was modified from the reference: Fares I, J Chagraoui, Y Gareau, et al. (2014). 
2) Harvest the stem cells, centrifuge at $300 \mathrm{~g}$ for 5 minutes, and remove the supernatant. Resuspend the cells in IMDM with $2 \%$ FBS, and count using Trypan blue.

3) Resuspend 500 cells in $100 \mu \mathrm{l}$ medium, add MethoCult H4034 Optimum 1 $\mathrm{mL}$ per well, transfer the mixture into 6 well plate.

4) Culture at $37^{\circ} \mathrm{C}, 5 \% \mathrm{CO}_{2}$ supplemented incubator for 14 - 16 days, count CFU-mix, CFU-GM, CFU-GEMM, BFU-E.

\subsection{HSC Functional Analysis in Vivo-Transplantation Study}

The NSG mice at aging of 8 - 12 weeks are prepared for HSC transplantation by feeding with water containing antibiotics. $1 \times 10^{5}$ of uncultured or expansed CD34+ cells are injected into the NSG mice sublethally irradiated (300 cGy) via tail vein. The mice were sacrifised 13 - 16 weeks after transplantation for assay of engrafment of human hematopoietic cells.

Blood cells and bone marrow (BM) cells from each mouse are analyzed for presense of cells expressing markers of human hematopoietic cells by flow cytometry. The markers usually include CD45, CD34, CD33, CD3, CD14, CD19, CD41 and CD71. For secondary transplantation, $2 \times 10^{6}$ of bone marrow cells from the primary receptient NSG mouse are transplanted into sublethally radiated NSG mouse. Blood cells and BM cells from mice 15 - 16 weeks after transplantation are collected to analyze the presence of human hematopoietic cells as above [1] [20].

The frequency of HSC in uncultured or expanded cells is analyzed by limiting dilution analysis (LDA). In each LDA experiment, mice are transplanted with 2000, 1000, 500 expanded cells. HSC frequency is calculated using L-Calc software (STEMCELL Technologies) and plotted using ELDA software (http://bioinf.wehi.edu.au/software/elda/). Poisson statistics is used to calculate the p-value for all LDA analyses. Differences in HSC frequencies are analyzed by the chi-square test. ${ }^{*} \mathrm{P}$ values $\leq 0.05$ are considered statistically significant [1] [6] [34].

\section{Conclusion}

HSCs derived from umbilical cord blood have advantages inaccessible and hematopoiesis capacity over HSCs derived from adult tissues. The limited number of UCB HSCs impedes its clinical application. Expansion ex vivo of UCB HSCs could overcome the obstacles. In general, there are three different types of division for a single HSC: symmetric renewal, asymmetric renewal and symmetric commitment. The strategy for expansion ex vivo of HSC aims to maintain symmetric renewal and avoid symmetric commitment. However, the mechanism underlying determination of HSC division fate remains unknown. We currently have no good way to harness HSC division fate in vitro. Most chemicals and growth factors which were applied in HSC ex vivo expansion, were identified through screening chemical library or recombinant growth factors based on in 
vitro and in vivo hematopoiesis capacity of the expanded HSC. The growth factors, including SCF, TPO and Flt-3L, are able to stimulate cell number increase by activating signaling pathways related to cell growth, but not able to maintain HSC stemness. Meanwhile, the chemical molecules, including SR-1, UM171, VPA, can maintain HSC stemness during HSC division by modifying metabolism or/and epigenetics pattern. Therefore, a few combinations of the identified growth factors with chemical molecules have been successfully applied in UCB HSC ex vivo expansion. But the process for optimization of the variable fumula compositions of different growth factors or of different chemical molecules or of the combinations growth factor with chemical molecule is necessary and time costuming, the optional mathematics models can benefit for guiding the optimization process. In this paper, we integrate the updates in ex vivo expansion of UCB HSC and provide an impact and practicable procedure from the protocol of isolation of original UCB HSC to the protocol of character identification of expanded UCB HSC.

\section{Conflicts of Interest}

The authors declare no conflicts of interest regarding the publication of this paper.

\section{Source of Support}

This research was supported by Logistic research project of china (aws17j007).

\section{References}

[1] Mantel, C.R., O’Leary, H.A., Chitteti, B.R., et al. (2015) Enhancing Hematopoietic Stem Cell Transplantation Efficacy by Mitigating Oxygen Shock. Cell, 161, 1553-1565. https://doi.org/10.1016/j.cell.2015.04.054

[2] Andrade, P.Z., Dos, S.F., Cabral, J.M., et al. (2015) Stem Cell Bioengineering Strategies to Widen the Therapeutic Applications of Haematopoietic Stem/Progenitor Cells from Umbilical Cord Blood. Journal of Tissue Engineering and Regenerative Medicine, 9, 988-1003. https://doi.org/10.1002/term.1741

[3] Wang, W., Fujii, H., Kim, H.J., et al. (2017) Enhanced Human Hematopoietic Stem and Progenitor Cell Engraftment by Blocking Donor T Cell-Mediated TNF $\alpha$ Signaling. Science Translational Medicine, 9, g3214.

https://doi.org/10.1126/scitranslmed.aag3214

[4] Saudemont, A. and Madrigal, J.A. (2017) Immunotherapy after Hematopoietic Stem Cell Transplantation Using Umbilical Cord Blood-Derived Products. Cancer Immunology, Immunotherapy, 66, 215-221. https://doi.org/10.1007/s00262-016-1852-3

[5] Ballen, K.K., Gluckman, E. and Broxmeyer, H.E. (2013) Umbilical Cord Blood Transplantation: The First 25 Years and Beyond. Blood, 122, 491-498. https://doi.org/10.1182/blood-2013-02-453175

[6] Gupta, R., Turati, V., Brian, D, et al. (2020) Nov/CCN3 Enhances Cord Blood Engraftment by Rapidly Recruiting Latent Human Stem Cell Activity. Cell Stem Cell, 26, 527-541. https://doi.org/10.1016/j.stem.2020.02.012 
[7] Kaur, K., Mirlashari, M.R., Kvalheim, G., et al. (2013) 3,4'-Dimethoxyflavone and Valproic Acid Promotes the Proliferation of Human Hematopoietic Stem Cells. Stem Cell Research \& Therapy, 4, 60. https://doi.org/10.1186/scrt208

[8] Delaney, C., Heimfeld, S., Brashem-Stein, C., et al. (2010) Notch-Mediated Expansion of Human Cord Blood Progenitor Cells Capable of Rapid Myeloid Reconstitution. Nature Medicine, 16, 232-236. https://doi.org/10.1038/nm.2080

[9] M-Reboredo, N., Díaz, A., Castro, A., et al. (2000) Collection, Processing and Cryopreservation of Umbilical Cord Blood for Unrelated Transplantation. Bone Marrow Transplant, 26, 1263-1270. https://doi.org/10.1038/sj.bmt.1702728

[10] Faivre, L., Couzin, C, Boucher, H., et al. (2018) Associated Factors of Umbilical Cord Blood Collection Quality. Transfusion, 58, 520-531.

https://doi.org/10.1111/trf.14447

[11] Ito, K. and Suda, T. (2014) Metabolic Requirements for the Maintenance of SelfRenewing Stem Cells. Nature Reviews Molecular Cell Biology, 15, 243-256. https://doi.org/10.1038/nrm3772

[12] Viny, A.D., Bowman, R.L., Liu, Y., et al. (2019) Cohesin Members Stag1 and Stag 2 Display Distinct Roles in Chromatin Accessibility and Topological Control of HSC Self-Renewal and Differentiation. Cell Stem Cell, 25, 682-696. https://doi.org/10.1016/j.stem.2019.08.003

[13] Calvi, L.M., Adams, G.B., Weibrecht, K.W., et al. (2003) Osteoblastic Cells Regulate the Haematopoietic Stem Cell Niche. Nature, 425, 841-846.

https://doi.org/10.1038/nature02040

[14] Morrison, S.J. and Scadden, D.T. (2014) The Bone Marrow Niche for Haematopoietic Stem Cells. Nature, 505, 327-334. https://doi.org/10.1038/nature12984

[15] Mendelson, A. and Frenette, P.S. (2014) Hematopoietic Stem Cell Niche Maintenance during Homeostasis and Regeneration. Nature Medicine, 20, 833-846. https://doi.org/10.1038/nm.3647

[16] Ema, H., Takano, H., Sudo, K., et al. (2000) In Vitro Self-Renewal Division of Hematopoietic Stem Cells. Journal of Experimental Medicine, 192, 1281-1288. https://doi.org/10.1084/jem.192.9.1281

[17] Yamazaki, S., Ema, H., Karlsson, G., et al. (2011) Nonmyelinating Schwann Cells Maintain Hematopoietic Stem Cell Hibernation in the Bone Marrow Niche. Cell, 147, 1146-1158. https://doi.org/10.1016/j.cell.2011.09.053

[18] Rossmanith, T., Schröder, B., Bug, G., et al. (2001) Interleukin 3 Improves the ex Vivo Expansion of Primitive Human Cord Blood Progenitor Cells and Maintains the Engraftment Potential of Scid Repopulating Cells. Stem Cells, 19, 313-320. https://doi.org/10.1634/stemcells.19-4-313

[19] Boitano, A.E., Wang, J., Romeo, R., et al. (2010) Aryl Hydrocarbon Receptor Antagonists Promote the Expansion of Human Hematopoietic Stem Cells. Science, 329, 1345-1348. https://doi.org/10.1126/science.1191536

[20] Fares, I., Chagraoui, J., Gareau, Y., et al. (2014) Cord Blood Expansion. Pyrimidoindole Derivatives Are Agonists of Human Hematopoietic STEM Cell Self-Renewal. Science, 345, 1509-1512. https://doi.org/10.1126/science.1256337

[21] Wagner, J.J., Brunstein, C.G., Boitano, A.E., et al. (2016) Phase I/II Trial of StemRegenin-1 Expanded Umbilical Cord Blood Hematopoietic Stem Cells Supports Testing as a Stand-Alone Graft. CELL STEM CELL, 18, 144-155. https://doi.org/10.1016/j.stem.2015.10.004

[22] Cohen, S., Roy, J., Lachance, S., et al. (2020) Hematopoietic Stem Cell Transplanta- 
tion Using Single UM171-Expanded Cord Blood: A Single-Arm, Phase 1-2 Safety and Feasibility Study. The Lancet Haematology, 7, e134-e145. https://doi.org/10.1016/S2352-3026(19)30202-9

[23] Haran, M. and Gross, A. (2014) Balancing Glycolysis and Mitochondrial OXPHOS: Lessons from the Hematopoietic System and Exercising Muscles. Mitochondrion, 19, 3-7. https://doi.org/10.1016/j.mito.2014.09.007

[24] Luchsinger, L.L., Strikoudis, A., Danzl, N.M., et al. (2019) Harnessing Hematopoietic Stem Cell Low Intracellular Calcium Improves Their Maintenance in Vitro. Cell Stem Cell, 25, 225-240. https://doi.org/10.1016/j.stem.2019.05.002

[25] Montgomery, D.C. (2017) Design and Analysis of Experiments. 9th Edition, Wiley, Hoboken.

[26] Ahmad, F., Ashraf, N., Zhou, R.B., et al. (2019) Optimization for Silver Remediation from Aqueous Solution by Novel Bacterial Isolates Using Response Surface Methodology: Recovery and Characterization of Biogenic AgNPs. Journal of Hazardous Materials, 380, Article ID: 120906. https://doi.org/10.1016/j.jhazmat.2019.120906

[27] Abdous, B., Sajjadi, S.M. and Mani, L.M. (2017) $\beta$-Cyclodextrin Modified Mesoporous Silica Nanoparticles as a Nano-Carrier: Response Surface Methodology to Investigate and Optimize Loading and Release Processes for Curcumin Delivery. Journal of Applied Biomedicine, 15, 210-218. https://doi.org/10.1016/j.jab.2017.02.004

[28] Ahmad, I., Yanuar, A., Mulia, K., et al. (2017) Optimization of Ionic Liquid-Based Microwave-Assisted Extraction of Polyphenolic Content from Peperomia pellucida (L) Kunth Using Response Surface Methodology. Asian Pacific Journal of Tropical Biomedicine, 7, 660-665. https://doi.org/10.1016/j.apjtb.2017.06.010

[29] Uddin, M.G., Allardyce, B.J., Leal, D., et al. (2020) Exfoliating B. mori Silk into High Aspect Ratio Nanofibrils Facilitated by Response Surface Methodology. International Journal of Biological Macromolecules, 164, 2389-2398. https://doi.org/10.1016/j.ijbiomac.2020.08.106

[30] Bezerra, M.A., Santelli, R.E., Oliveira, E.P., et al. (2008) Response Surface Methodology (RSM) as a Tool for Optimization in Analytical Chemistry. Talanta, 76, 965-977. https://doi.org/10.1016/j.talanta.2008.05.019

[31] Rishad, K.S., Rebello, S., Nathan, V.K., et al. (2016) Optimised Production of Chitinase from a Novel Mangrove Isolate, Bacillus pumilus MCB-7 Using Response Surface Methodology. Biocatalysis and Agricultural Biotechnology, 5, 143-149. https://doi.org/10.1016/j.bcab.2016.01.009

[32] Shahbazi, N. and Zare-Dorabei, R. (2019) A Novel "Off-On” Fluorescence Nanosensor for Sensitive Determination of Sulfide Ions Based on Carbon Quantum Dots and Gold Nanoparticles: Central Composite Design Optimization. Microchemical Journal, 145, 996-1002. https://doi.org/10.1016/j.microc.2018.12.022

[33] Chagraoui, J., Lehnertz, B., Girard, S., et al. (2019) UM171 Induces a Homeostatic Inflammatory-Detoxification Response Supporting Human HSC Self-Renewal. PLoS ONE, 14, e224900. https://doi.org/10.2139/ssrn.3381950

[34] Wilkinson, A.C., Ishida, R., Kikuchi, M., et al. (2019) Long-Term ex Vivo Haematopoietic-Stem-Cell Expansion Allows Nonconditioned Transplantation. Nature, 571, 117-121. https://doi.org/10.1038/s41586-019-1244-X

[35] Csaszar, E., Kirouac, D.C., Yu, M., et al. (2012) Rapid Expansion of Human Hematopoietic Stem Cells by Automated Control of Inhibitory Feedback Signaling. Cell Stem Cell, 10, 218-229. https://doi.org/10.1016/j.stem.2012.01.003 